\title{
About Place Cells and Grid Cells
}

\author{
Christos H. Papadimitriou $\left.{ }^{(}\right)$ \\ Computer Science, Columbia University, New York, NY, USA \\ christos@columbia.edu
}

\begin{abstract}
Almost half a century ago, it was discovered that there are special neurons in the hippocampus of mammals that are activated every time the animal is in a particular place within its familiar surroundings [2]. If you let the animal roam freely near this place (say, if its path is the black line in Fig. 1), then this cell will fire at the red dots. These neurons are called place cells, and they seem to be part of the mental map animals need to get around in the world: they navigate a familiar terrain by going from one place cell to the next.
\end{abstract}

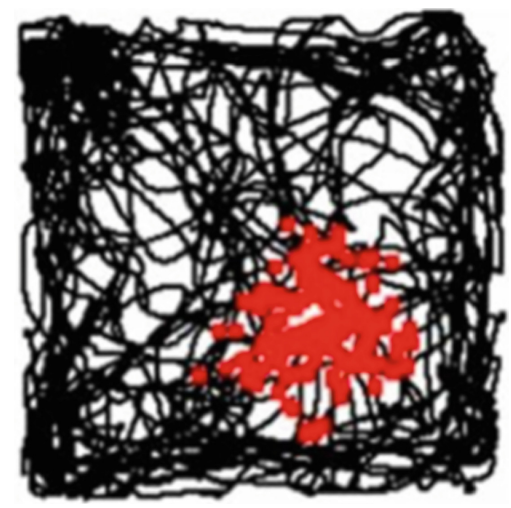

Fig. 1. A place cell fires when the animal is at or near a particular place (image from http://www.ucl.ac.uk/jefferylab/research.) (Color figure online)

Do we have place cells too? All mammals seem to have them, and I doubt that the capability was lost in evolution. Neuroscientists indeed have started spotting human place-like cells. But, for some reason unrelated to scientific evidence or insight, I like to believe that, in our brains, place cells have an elevated mission. That they may also record non-spatial loci particular to our species. Like the downhill pace when the murderer is about to be revealed in the mystery novel we are reading, or the mental labyrinth we inhabit when our algebra is not working. Or the sublime flash when we are starting to understand the gist of a proof that we have been wading through. Or the quiet bliss when - a faint smile in our lip - we are reminiscing about a friend.

My encounters with Juraj have been too few and far across, but I remember them vividly and fondly. A gentle and affable man, cordial and genuine in manner, quietly witty in conversation, a mensch who listens to you with shiny eyes

(C) Springer Nature Switzerland AG 2018

H.-J. Böckenhauer et al. (Eds.): Hromkovič Festschrift, LNCS 11011, pp. 637-640, 2018.

https://doi.org/10.1007/978-3-319-98355-4_36 
and understated smile - a smile that tells you "I do have something intelligent to say, but carry on." A meeting with Juraj is a boon to the mood and the intellect - and it almost always includes a hike. Because Juraj wants to share with his friends some of his favorite place cells.

Meanwhile, back to the hippocampus: during the last decade and a half the plot thickened-literally!

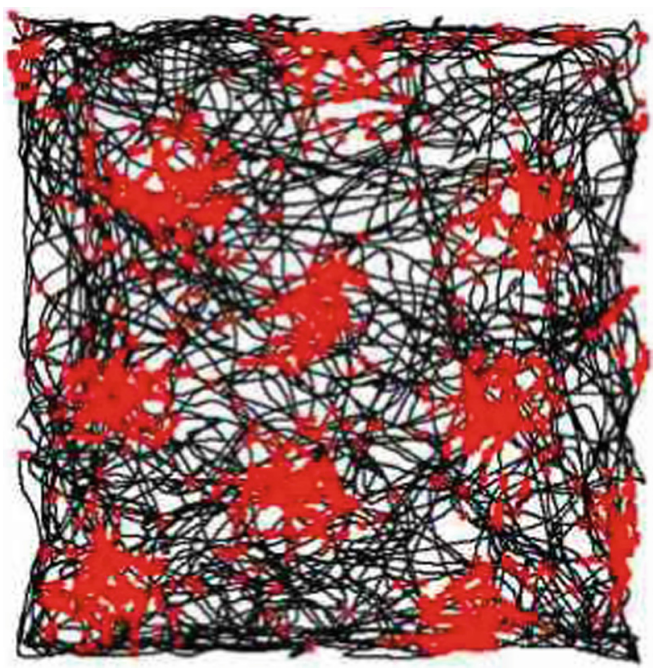

Fig. 2. A grid cell fires when the animal is at or near any one of the points in a triangular grid spanning a familiar domain (image from http://www.ucl.ac.uk/news/ news-articles/1001/10012001.)

Grid cells are not in the hippocampus, but they live nearby in its gateway, another tiny and ancient piece of our brain called the entorhinal cortex, see [2] for a description. Note that Fig. 2 shows the response of one cell; the same neuron fires when the animal is close to any one of the vertices of this network.

At this point one has to stop and admit that this is remarkable. I like to think that the first geometry conceived by humans was discrete, not unlike the one of grid cells. The primitive human probably first experienced mathematics when she found a quiet moment to admire - from her fully erect posture - the starry sky. Much later we settled in places and cultivated the land and had to divide it, and so the Pythagoreans gave geometry its laws and stories. Ultimately, a man in Alexandria codified them all in thirteen books - of which the seventh, incidentally, contains the first piece of code ever written: "Keep subtracting the smaller from the larger until they're equal', , because for Euclid integers were geometry too, lengths of rope. About twenty centuries went bysome good, most bad - and finally a Frenchman whose Latin name was Cartesius decided to go the opposite way: he found a sensible framework for codifying 
geometry with numbers. It took two more centuries of exploring the new possibilities, until - the horror! - geometries were stumbled upon that do not adhere by Euclid's axioms. These paradoxical concoctions puzzled and frustrated Immanuel Kant, who believed that geometry is about the only domain in science that cannot be relative, because our brain is embedded in it. And now, another two centuries later, we suddenly discover that brains have their own geometry - and it ain't ours.

But why this particular geometry? In neuroscience, any cell that responds to a stimulus (in this case, the animal's position) is characterized by its tuning curve, a mathematical function mapping the space of all stimuli (here, the unit square) to the positive reals. If the stimulus is $x$, then $f(x)$ is, roughly speaking, the probability that the neuron will fire upon sensing this stimulus. In this particular case, the tuning curve of grid cells is a surface in $3 \mathrm{D}$ that brings to mind a terrain with steep bell-shaped hills (capped by red peaks) arranged in a triangular grid, interleaved with concave troughs (the blacker places in the figure), also triangularly arranged. Why this tuning curve? What is the origin and logic of grid cells?

The dominant hypothesis about the function of grid cells is that they help with path integration. One idea (and there are many others) is that they help calculate the animal's position by somehow measuring and integrating elementary displacements (the integration is presumably done by another system of neurons, somewhere else). The measurement of the elementary displacements is done by measuring differences in the firing rate of the grid cell. The question is, does this theory justify the bizarre shape? If we were to design a neuron that measures displacement this way, which is the function $f$ that optimizes the accuracy of this measurement?

This is a hard problem, and so we perform a familiar maneuver: we solve an easier, one-dimensional problem, and in fact one whose geometry has no edge effects. Suppose that the domain of the animal is the circumference of a circle; what is the tuning curve that will yield the most accurate measurements? This simpler question has an interesting answer (see Fig. 3).

The optimum tuning curve is a one-dimensional grid cell! Ergo, periodic tuning curves do seem to make sense in the context of path integration. The details of the derivation can be found in [1], but the idea is roughly this: First, you postulate that there is a population of $n$ cells that measure displacement, and that their tuning curves, denoted $f_{i}(x)$ where $x$ is now an angle, are shifts of one another by multiples of $\frac{2 \pi}{n}$-notice that these tuning curves will eventually be proved the same. Measurement accuracy is best captured in this context by the Fisher information, a quantity which, through the Cramér-Rao theorem, bounds the variance of measurement. And it is known that in this case the Fisher information is given by the formula $\dot{f}(x)^{T} C^{-1} \dot{f}(x)$, where $\dot{f}(x)$ is the vector of the derivatives of the tuning curves of the $n$ neurons at the angle $x$, and $C$ is the matrix of the pairwise correlations of the measurement errors between the $n$ neurons. So, this is the quantity we need to maximize under a "bounded power" constraint, which says that the length of the vector $\dot{f}(x)$ is at most one. 


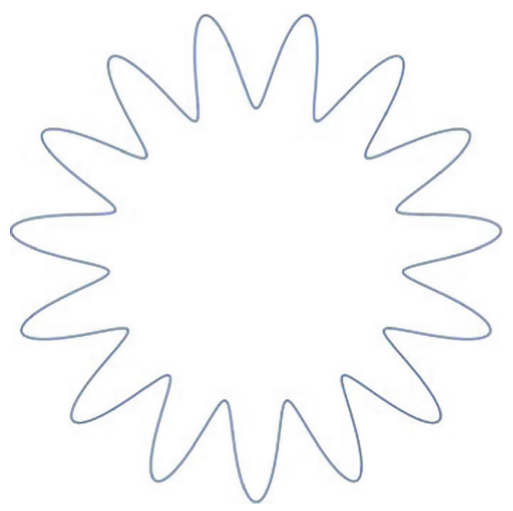

Fig. 3. The optimal one-dimensional tuning curve.

Now, $C$ is both symmetric and circulant, due to the symmetries of the situation, and the Courant-Fischer theorem tells us that in symmetric matrices the unit vector that maximizes $\dot{y}^{T} C^{-1} y$ is the eigenvector of $C$ corresponding to the smallest eigenvalue (the largest eigenvalue of $C^{-1}$ ). This eigenvector is a real-valued sinusoid, because the matrix is circulant, which concludes the sketch.

Does this exercise help us understand the logic behind the tuning curve of the two-dimensional grid cell? As explained in [1], it does, but only very little. The tuning curve of the two-dimensional grid cell is easily seen to be the product of two sinusoids (plus one, of course), and once we assume a product form of two independently optimized factors, the three-dimensional grid cells we know are the solution - at least on the torus. But too many assumptions were used.

There is a little epilogue to the story: how about three dimensions? Experiments with bats reveal that, indeed, they do embed their familiar flying space into a three-dimensional grid, whose structure is still a bit of a mystery (for a plausible prediction see the last section of [1]). And bats are not the only mammals who live in three dimensions, there are dolphins in the world, and walruses, and whales.

And of course climbers like my friend Juraj, to whom I am bestowing my warmest feelings and thoughts and my best wishes for a happy birthday.

\section{References}

1. Papadimitriou, C.H.: On the optimality of grid cells. Technical report (2018). https://arxiv.org/abs/1606.04876

2. Moser, E.I., Kropff, E., Moser, M.-B.: Place cells, grid cells, and the brain's spatial representation system. Annu. Rev. Neurosci. 31(1), 69-89 (2008) 